\title{
ARTIFICIAL INTELLIGENT SYSTEM FOR MEASUREMENT OF HARMONIC POWERS
}

\author{
Jovitha Jerome* and P. Vinoth \\ Department of Electrical and Electronics Engineering, \\ P.S.G. College of Technology, Tamilnadu, India
}

Received 08 June 2006

\begin{abstract}
The importance of the electric power quality (PQ) demands new methodologies and measurement tools in the power industry for the analysis and measurement of the basic electric magnitudes necessary. This paper presents a new measurement procedure based on neural networks for the estimation of harmonic amplitudes of current/voltage and respective harmonic powers. The measurement scheme is built with two neural network modules. The first module is an adaptive linear neuron (ADALINE) that is the kernel part of estimation of complex harmonic coefficients of the current/voltage. The second module is feedforward neural network that obtains the harmonic active/reactive powers. In order to perform digital simulation the Feedforward and Adaline neural network tools were developed in LabVIEW. This measurement algorithm was tested for the practical cases and found to be robust, computationally fast and efficient.
\end{abstract}

Keywords: Adaptive estimation techniques, artificial neural networks (ANNs), harmonics, and measurements.

\section{INTRODUCTION}

The widespread applications of electronically controlled loads have increased the harmonic distortion in power system voltage and current waveforms in the last few decades. The largescale presence of single-phase and three-phase nonlinear loads in power systems makes necessary a system monitoring of harmonic powers and unbalanced current/voltage. The problem of estimating the amplitudes, phase angles and frequencies of sinusoidal signals from noisy and distorted data has received considerable attention recently due to proliferation of power electronic loads in electric power networks. Further the estimation of basic parameters of voltage and current signals is a prerequisite for evolving suitable protection and control strategies of power networks. Power quality issues are very much dependent on the RMS amplitudes of these waveforms and hence suitable analysis methodologies and measurement tools are assuming importance in the power industry. The identification of harmonics produced by the nonlinear loads and the determination of the degree of voltage and current unbalance takes part in the evaluation requirements of electric waveform quality [1]. Thus, the importance of the electric power quality (PQ) makes having new methodologies for the analysis and measurement of the basic electric magnitudes in these conditions necessary.

The current methods can be categorized into two classes, the traditional methods and non linear

${ }^{*}$ Corresponding author e-mail: jjm@eee.psgtech.ac.in 
adaptive method. The traditional methods are DFT and linear filters, and the newer methods are nonlinear adaptive techniques using neural networks. The traditional techniques are popular because they are well-known, easy to implement and require very little computational power. The conventional filter and Fourier transform techniques give often results of insufficient precision because of signal interference or numerical effects (leakage, aliasing or picket-fence effect), especially in such cases where the period of the frequency component to be detected is not a integer multiple of the measurement sampling interval. Furthermore, for dynamic control or monitoring purpose a fast harmonic detection is essential to ensure a good performance. In many situations it is even desirable to know or to predict in advance the trajectory (or the parameters) of these varying harmonic components in time-domain rather than in frequencydomain. An efficient on-line estimation method for such applications is the Kalman filter technique [2]. However, the performance of a standard recursive Kalman algorithm is strongly dependent on the a priori information of the process and measurement noise which is either unknown or is known only approximately in practical situations.

The use of neural networks appears as alternative method for tracking of harmonics in power line. New parallel algorithms for estimation of parameters of sine wave contaminated by noise are proposed. The problem of estimation is formulated as an optimization problem and solved by using the gradient descent method. Algorithms based on the least absolute value, the leastsquares and the minimax (Chebyshev) criteria are developed and compared [3]. A new algorithm for the estimation of parameters of voltage or current waveform of power networks contaminated by noise is proposed. The problem of estimation is formulated by using an adaptive neural network consisting of linear adaptive neurons called adaline. The learning parameters of the adaline are adjusted to force the error between the actual and desired outputs to satisfy a stable difference error equation, rather than to minimize an error function [4].

A new algorithm along new architecture of analogue neuron-like adaptive processors for online estimation of parameters of sinusoidal signals, which are distorted by higher harmonics and corrupted by noise [5]. Three adaptive linear neurons are used to tackle the desired three phase filter current templates [6]. A new control method for Active Power Filters (APF) using Neural Networks is presented. It's a Pulse Width Modulation control (PWM) designed with two blocks that include Neural Networks. The first one includes adaptive networks that estimate the reference compensation current adaptive linear neurons (Adaline) were used. The second one includes a feedforward network that works as a hysteresis band comparator; this network is multilayer and it has been trained by a backpropagation algorithm [7]. The adaptive and feedforward neural networks techniques have been systematically explored for symmetrical components estimation. Thus, an original method for the measurement of three-phase current/voltage harmonic components and harmonic powers in unbalanced systems is presented. The proposed technique is based on an estimation of the instantaneous time phasors using the ANN with a reduced computational cost.

\section{Objective}

The two primary objectives of this paper are:

1) To create a technique that is able to measure and extract harmonically-related sinusoids in stationary and nonstationary situations with high noise immunity.

2) Provide a straightforward technique for implementation of power quality monitoring devices for harmonics measurement.

\section{Methodology}

This paper proposes a new approach based on the application of neural networks to the estimation of the magnitude and phase of the voltage/current of individual harmonics. Fig. 1 
presents the general scheme of the neural measurement system (NMS). The two modules are distinguished. Module 1 gives the complex coefficients of harmonics present in the voltagelcurrent, the expansions is built by a set of adaptive neurons with an online training process (ADALINE). Module 2 (a feedforward ANN) permits to obtain the harmonic powers (activelreactive) of each individual harmonic.

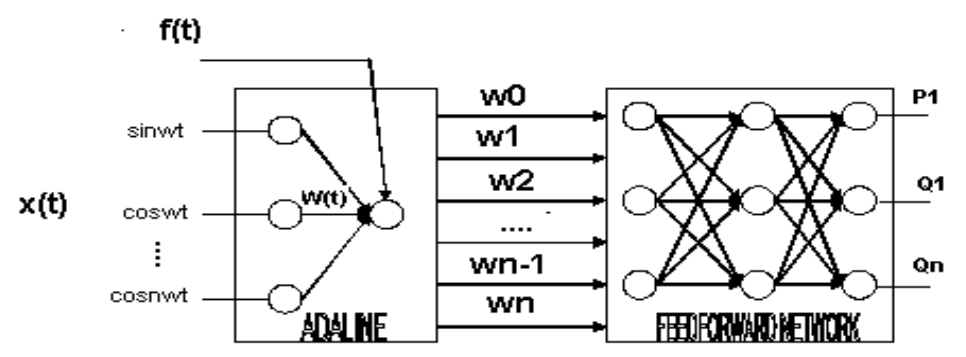

Fig. 1: The neural measurement system.

\section{STATEMENT OF THE PROBLEM}

Consider the following sinusoidal signal (currentlvoltage) distorted by a dc exponential component and random noise.

$$
y(t)=A \sin (\omega t+\varphi)+A_{d c} e^{-\alpha_{d c} t}+\in(t)
$$

in which, $A, A_{d c}$ are unknown amplitudes

$$
\omega=2 \pi f \text { is unknown angular frequency }
$$

Further instead of fixed amplitude and phase of the sinusoidal signal described in equation (1), the time variations of the phase voltage at a given power network node is described by the following equation (2).

$$
y(t)=A(t) \sin (\omega(t)+\varphi(t))+\in(t)
$$

To estimate the amplitude $A(t)$, phase angle $\varphi(t)$ and $A_{d c}$ and $\alpha_{d c}$ of the decaying dc component, equation (2) is rewritten using first two terms of the Taylor series expansion for the decaying dc component.

$$
y(t)=X_{a}(t) \cos \omega t+X_{b}(t) \sin \omega t+A_{d c}-A_{d c} \alpha_{d c} t
$$

where

$$
A(t)=\sqrt{X_{a}^{2}(t)+X_{b}^{2}(t)}, \varphi(t)=\tan ^{1}\left(X_{b}(t) / X_{a}(t)\right)
$$

Further equation (3), (4) can be written in a discrete form as (5)

$$
y(k)=X_{a}(k) \cos \theta+X_{b}(k) \sin \theta+A_{d c} \quad A_{d c} \alpha_{d c} t
$$

where

$$
\Delta t=\frac{2 \pi}{\omega N_{s}}, \theta=\frac{2 \pi k}{N_{s}}
$$

the active and reactive power can be estimated in general using (6) 


$$
\begin{aligned}
& P_{n}=V_{n} I_{n} \cos \varphi \\
& Q_{n}=V_{n} I_{n} \sin \varphi
\end{aligned}
$$

where

$V_{n}=$ amplitude of nth harmonic

$I_{n}=$ amplitude of nth harmonic

$\varphi=$ phase angle between voltage and current

There exists a need for an on-line algorithm that can satisfy two simultaneous requirements:

(a)directly providing estimates of the parameters $X_{a}, X_{b}, A_{d c}$ and $\alpha_{d c}$ from the given data samples $y(k)$ obtained from the measurement.

(b) provide improved estimates as updated sample are received.

In other words, the estimated parameters need to be more accurate with each update for the stationary signal, for nonstationary signals, the estimates are required to track the time varying amplitude $A(t)$ and phase angle $\varphi(t)$.Finally, the active power and reactive power of each harmonic can be calculated by means of current/voltage-symmetrical components phasors.

\section{NEURAL NETWORK FOR SIGNAL TRACKING AND POWER ESTIMATION}

A neural network is a parallel, distributed information processing structure composed of basic processing elements known as neurons. Artificial neural networks (ANNs) are networks consisting of man-made models of biological neurons, implemented in either hardware or software. In neurons, input signals are carried across synapses which weight the individual signals, and into the cell body which combines the weighted signals; the new signal is output through an axon which can, in turn, connect to one or more synapses. In this single-neuron ADALINE model is used, the combination of input signals is accomplished through a linear weighted sum of the input signals; however, other non-linear combinations are possible.

The ADALINE (ADAptive LInear NEuron networks) network discussed in this paper is similar to the perceptron, but their transfer function is linear rather than hard-limiting. This allows their outputs to take on any value, whereas the perceptron output is limited to either 0 or 1 . Both the ADALINE and the perceptron can only solve linearly separable problems. However, here we will make use of the LMS (Least Mean Squares) learning rule, which is much more powerful than the perceptron learning rule. The LMS or Widrow-Hoff learning rule minimizes the mean square error and, thus, moves the decision boundaries as far as it can from the training patterns. Linear networks that are adjusted at each time step based on new input and target vectors can find weights and biases that minimize the network's sum-squared error for recent input and target vectors. Networks of this sort are often used in error cancellation, signal processing, and control systems. The pioneering work in this field was done by Widrow and Hoff, who gave the name ADALINE to adaptive linear elements.

\subsection{Linear neuron model}

A linear neuron with $\mathrm{n}$ inputs is shown in Fig. 2. To build an adaline neural network for this particular application, several processing elements or neurons are arranged in a particular fashion. Figure 2 shows the linear model of an artificial neuron. It basically consists of four different components: 


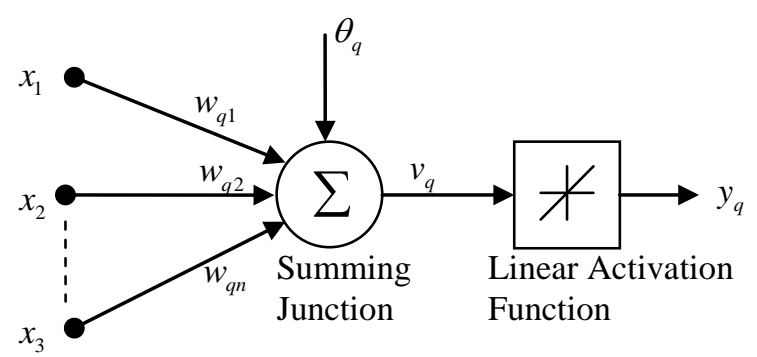

Fig. 2: Linear neuron model.

(i) There is a set of synapses with associated synaptic weights. The continuous valued input to the synapses is a vector signal $x \in \mathfrak{R}^{n x 1}$, with the individual vector component given as $x_{j}$, for $\mathrm{j}=1,2, \ldots \mathrm{n}$, that is $x=\left[x_{1}, x_{2} \ldots x_{n}\right]^{T}$.Therefore each vector component $x_{j}$ is input to the $\mathrm{j}^{\text {th }}$ synapses and connected to neuron $\mathrm{q}$ through synaptic weight $\mathrm{w}_{\mathrm{qj}}$. (ii) The threshold or bias $\theta$ is usually externally applied and exactly acts as weight on a connection from a unit whose activation is always one. Increasing the bias increases the net input to the unit. (iii) The summing device act to add all the signals that are broadcast into the adder; that is, each input is multiplied by the associated weight and then summed. All the operations up to including the output of the adder constitute a linear combination, because the output of the adder is a linear combination of the inputs to the synapses. (iv)The activation function serves to limit the amplitude on the neuron output is a linear function in this case. The Widrow-Hoff rule can only train single-layer linear networks. This is not much of a disadvantage; however, as single-layer linear networks are just as capable as multilayer linear networks. For every multilayer linear network, there is an equivalent single-layer linear network.

\subsection{Input-output modeling for tracking using adaline neural network}

The purpose of module 1 (Adaline) of Neural Measurement System (NMS) is to present a new algorithm for fast tracking of voltage and current phasors using an adaptive neural network consisting of linear adaptive neurons called Adaline. The structure is based on the early work of B.Widrow in the 1960s [8] and has been widely applied in neural networks, signal processing and many other areas. A generalized weight adaptation algorithm for the adaptive linear combiner is used to arrive at the magnitude and phase of the voltage or current phasor. In this algorithm, the learning parameters are adjusted to force the error between the actual and desired outputs to satisfy a stable difference error equation, rather than to minimize an error function. This approach allows one to better control the stability and speed of convergence by appropriate choice of parameters of the error difference equation. The algorithm presented in paper is an adaptive one and is based on the assumption that the frequency of the fundamental voltage or current phasor is known apriori. A Lyapunov function based learning parameter is used to provide fast convergence and noise rejection. Several numerical examples along with real-time test results are given to validate its performance in the presence of decaying dc components and noise.

The regression input vector for this network is written as (7)

$$
X^{T}(k)=[1 \sin \theta \cos \theta]
$$

where $T=$ transpose of a quantity

However, if no dc component is required to be tracked, the input regressor becomes 


$$
X^{T}(k)=[\sin \theta \cos \theta]
$$

For tracking several periodic components (with multiple periods of the fundamental), the equation is given as (8)

$$
X^{T}(k)=[1 \sin \theta \cos \theta \sin 2 \theta \ldots \ldots . \cos m \theta]
$$

The block diagram of an adaline is shown in Fig. 3

And the weight matrix $W^{T}$ required for the equation 5 is obtained using any of the below methods.

\section{Method 1}

The Widrow-Hoff adaptation algorithm often referred to as the Widrow-Hoff delta rule, can be used to adapt the adaline's weight vector. The delta rule which minimizes the mean square error between the signal sample $y(k)$ and the output $\hat{y}(k)$ over all $k$, can be written as (9)

$$
W(k+1)=W(k)+\frac{\alpha e_{k} X_{k}(t)}{\lambda+X_{k}^{T}(t) X_{k}(t)}
$$

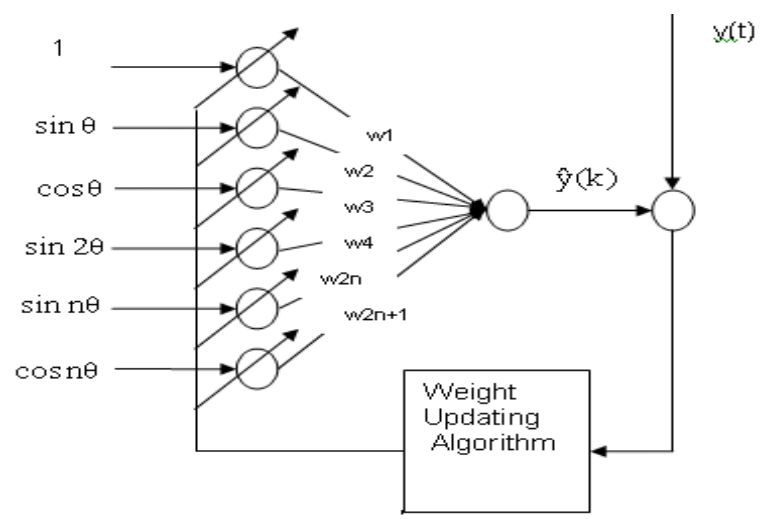

Fig. 3: Block diagram of adaline network for signal tracking.

where

$k=$ time index or sample number

$W(k)=\left[W_{l}(k) W_{2}(k) W_{3}(k) W_{n}(k)\right]^{T}$ is the weight vector time $k$,

$e(k)=y(k)-\hat{y}(k)$ is the error at time $k, \hat{y}(k)=$ estimated signal sample, given by (10)

$$
\hat{y}(t)=W^{T} \bullet x(t)=\left[X_{1} Y_{1} \ldots \ldots X_{N} Y_{N}\right] \cdot\left[\begin{array}{l}
1 \\
\operatorname{sinn} \omega_{1} t \\
\cos n \omega_{1} t \\
\ldots \ldots \\
\sin N \omega_{1} t \\
\cos N \omega_{1} t
\end{array}\right]
$$


$\alpha=$ reduction factor (learning parameter) and $\lambda$ is a constant chosen to be close to zero and is only included to avoid division by zero.

After the weight vector converges to a constant value $W_{0}$, the weight vector yields the signal parameter as

$W_{0}=\left[\begin{array}{lll}A_{d c} & X_{a} & X_{b}\end{array}\right]^{T}$ the amplitude and phase of the currentlvoltage phasor in general are obtained from the weight vector as (11)

$$
A=\sqrt{W_{2 n-1}(k)^{2}+W_{2 n}(k)^{2}} \text { and } \varphi=\tan ^{-1}\left\{W_{2 n}(k) / W_{2 n-l}(k)\right\}
$$

for fundamental voltage is given as (12),

$$
A=\sqrt{W_{I}(k)^{2}+W_{2}(k)^{2}} \text { and } \varphi=\tan ^{-1}\left\{W_{2}(k) / W_{I}(k)\right\}
$$

\section{Method 2}

This algorithm for the weight adaptation of adaline produces a fast convergence and introduces nonlinearity to the learning technique. The weight vector of the adaline is adapted as (13)

$$
W(k+1)=W(k)+\frac{\alpha e(k) \theta(k)}{\lambda+X^{T}(k) \theta(k)}
$$

where $\theta(k)$ is chosen as a SGN function given by (14)

$$
\theta(k)=\left[\begin{array}{lll}
1 & S G N(\sin \theta) \quad S G N(\cos \theta)
\end{array}\right]
$$

where $\operatorname{SGN}\left(x_{i}\right)=\left\{\begin{array}{cll}+1 & \text { if } & x_{i}>0 \\ -1 & \text { if } & x_{i}<0\end{array}\right.$

The learning rate $\alpha$ is kept between 0.1 and 2 for fast convergence. However, an adaptive variation of $\alpha$ is described for providing fast convergence and noise rejection for the neural estimation algorithm. Lyapunov function based $\alpha$ adaptation is attempted in this paper. A small $\alpha$ is required for a periodic sinusoidal waveform with constant peak amplitude and a large $\alpha$ for a waveform corrupted by distortions and noise. An alternative method for tuning the learning parameter $\alpha$ is derived in this paper by using Lyapunov function based formalism. The Lyapunov energy function is widely used in control system for providing stability and fast convergence of the system oscillations to their steady state values. A Lyapunov function $y(k)$ (15) is thus defined as

$$
V(k)=e^{2}(k)+c_{1}(e(k))^{2}
$$

and $c_{1}>0$.

The change in the Lyapunov function $V(k)$ is calculated as (16)

$$
\Delta V(k)=V(k)-V(k-1)
$$

The value of the learning parameter $\alpha(k)$ is chosen from (17), (18)

$$
\alpha(k)=\alpha_{0}+c_{2} V(k) \quad \text { for } \quad \Delta V(k)>0
$$




$$
\alpha(k)=\alpha_{0}-c_{2} V(k) \quad \text { for } \quad \Delta V(k)<0
$$

and $c 2>0$.

The value of $\alpha$ for the neural estimator is constrained between $0.1<\alpha(k)<1.5$ for optimum results. Also the values of $c_{1}, c_{2}$ are chosen in this paper as $c_{1}=0.2, c_{2}=0.2$ and $\lambda=0.01$

\subsection{Operation of feedforward neural network in neural measurement system}

The coefficients obtained from the first module (Adaptive Neural Network) are the inputs of the second module made of a feedforward neural network. The inputs number is 82 corresponds to current and voltage, the number of the output is 41 , which corresponds to active and reactive powers of the 20 harmonics, and the dc component. This architecture makes it possible to decrease the complexity of the network and increase the parallelism and computation speed. This election has a justification due to (6), where there is no dependence between the desired results for symmetrical components of each harmonic and the other harmonics. The first subnetwork built for calculating the symmetrical components through linear neurons due to the linearity. The power system was simulated with the LabVIEW to obtain the sets of inputs/outputs data essentials for the offline training. The training process of this block was realized following an equal method to that used with the first block, and also, it was as quick as that.

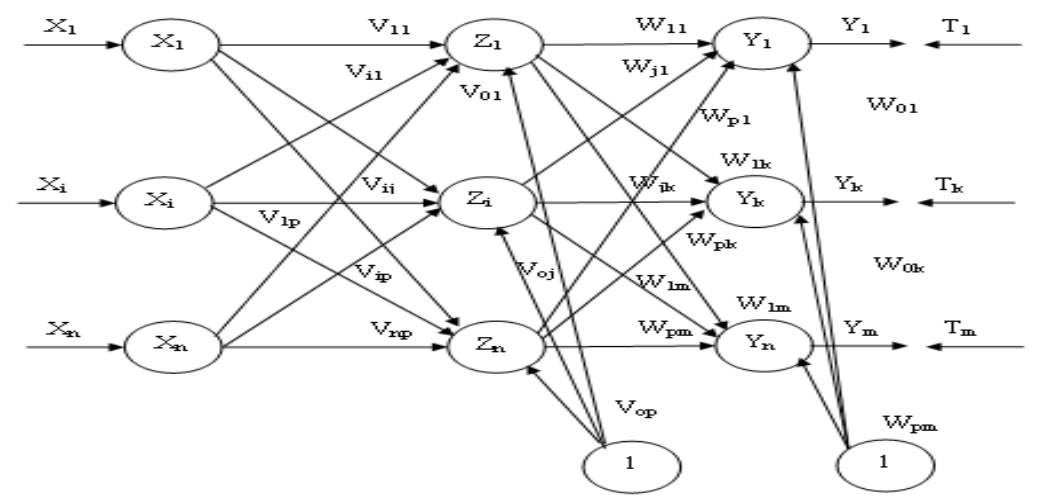

Fig. 4: Architecture of feed forward back propagation network.

The training method most commonly used for the feedforward network is the backpropagation training algorithm (Fig. 4). The network is initially untrained with selected random weights. The initial output pattern is compared with the desired output pattern, and the weights are adjusted by the algorithm until the error becomes small enough. The training process is carried out by means of a program that uses a large number of input/target data. The training input/target data can be derived from a simulation or from experimental results. Here the network is trained for obtaining the symmetrical components of voltagelcurrent and the harmonic active and reactive power form the weight matrix of adaline block. The table 1 shows the training details for power calculation using the feedforward neural network. The optimized value of hidden neuron found to be 10 shown bold in the table.

The parameters for power calculation are

Number of input unit $=81$

Number of output unit $=41$

Number of iteration $=4000$

Number of training pattern $=10$ 
Table 1: Comparison of training details for power calculation block.

\begin{tabular}{llll}
\hline Number of hidden unit & Learning rate & Total error & Simulation time(min) \\
\hline $\mathbf{1 0}$ & $\mathbf{0 . 1}$ & $\mathbf{0 . 5 5}$ & $\mathbf{8 3}$ \\
15 & 0.1 & 0.8179 & 117 \\
20 & 0.1 & 0.91 & 171 \\
\hline
\end{tabular}

\section{SIMULATION RESULTS}

In order to check the validity and performance of the proposed algorithm, numerical experimentation on the simulated waveforms has been carried out using LabVIEW software package. The simulations fully confirmed the correctness of the presented approach. The neural estimation algorithm is initialized by finding an optimized weight vector starting from a random set and minimizing an integral error square criterion. A sample rate of 81 based on the $50 \mathrm{~Hz}$ frequency is chosen for the estimation of signal amplitude and phase for all the studies. However, an optimized weight vector for waveform tracking is not a strict requirement when the learning parameter $\alpha$ is varied using a Lyapunov type of error function. The illustrative results to show the accurate tracking capability of the neural estimator is shown below for this test case.

Test Case: The following sinusoidal signal corrupted by decaying dc component, harmonics and random noise is chosen:

$$
\begin{aligned}
& v(t)=1.5 \sin \left(\omega t+29.3^{\circ}\right)+0.068 \sin \left(5 \omega t+141.6^{\circ}\right)+0.017 \sin \left(7 \omega t+66.2^{\bullet}\right)+ \\
& 0.022 \sin \left(11 \omega t-99.4^{\circ}\right)+0.024 \sin \left(13 \omega t-179.2^{\bullet}\right)+0.012 \sin \left(17 \omega t-1.3^{\circ}\right)+ \\
& 0.1 \exp (-5 t) \\
& i(t)=1 \sin \left(\omega t+60^{\circ}\right)+0.02 \sin \left(5 \omega t+141.6^{\bullet}\right)+0.017 \sin \left(7 \omega t+66.2^{\bullet}\right)+ \\
& 0.022 \sin \left(11 \omega t-99.4^{\circ}\right)+0.24 \sin \left(13 \omega t-179.2^{\circ}\right)+0.012 \sin \left(17 \omega t-1.3^{\circ}\right)+ \\
& 0.7 \exp (-5 t)
\end{aligned}
$$

The above waveform (19) pertains to an industrial load described in reference [4] and the exponentially decaying dc component and noise are added to test the new algorithm. Figure 5 and Fig. 6 show the amplitude and phase of the fundamental component, $5^{\text {th }}$ harmonic and $13^{\text {th }}$ harmonics, adaptive parameter, error, output and dc magnitude respectively.

The result shown in Fig. 5 is estimated using modified Widrow-Hoff method (method1) of weight updation. The adaptive parameter $\alpha$ is fixed value chosen to be 0.6 and $\lambda$ is fixed to small value in order to avoid divide by zero error. Figure 6 shows the results obtained with adaptive $\alpha$. In this for estimating the amplitude, and phase of the distorted signal, a SGN function is used for the weight adaptation. The initial value of $\alpha$ is chosen as 0.6 and is adapted using Lyapunov function during the estimation process as described in the Method 2. The variation of $\alpha$ is also shown in this figure. From the figure it is observed that in the presence decaying dc, harmonics and noise the tracking of the fundamental component is achieved in just about one cycle $(0.02 \mathrm{sec}$.

It is observed from Fig. 6 that the neural estimator with adaptive learning rate $\alpha$ produces a faster convergence and noise rejection in the presence of decaying dc component and random noise. However, without dc and random noise signal, the performances of all the estimators are similar and convergence is achieved in less than one cycle. 
Feedforward neural network after training for calculation of active and reactive power is tested. The weight vector obtained from the adaline neural network is given to feedforward neural network for active and reactive power calculation. The Fig.7 shows the active and reactive powers of fundamental, $5^{\text {th }}$ harmonic, $7^{\text {th }}$ harmonic, and $13^{\text {th }}$ harmonic.
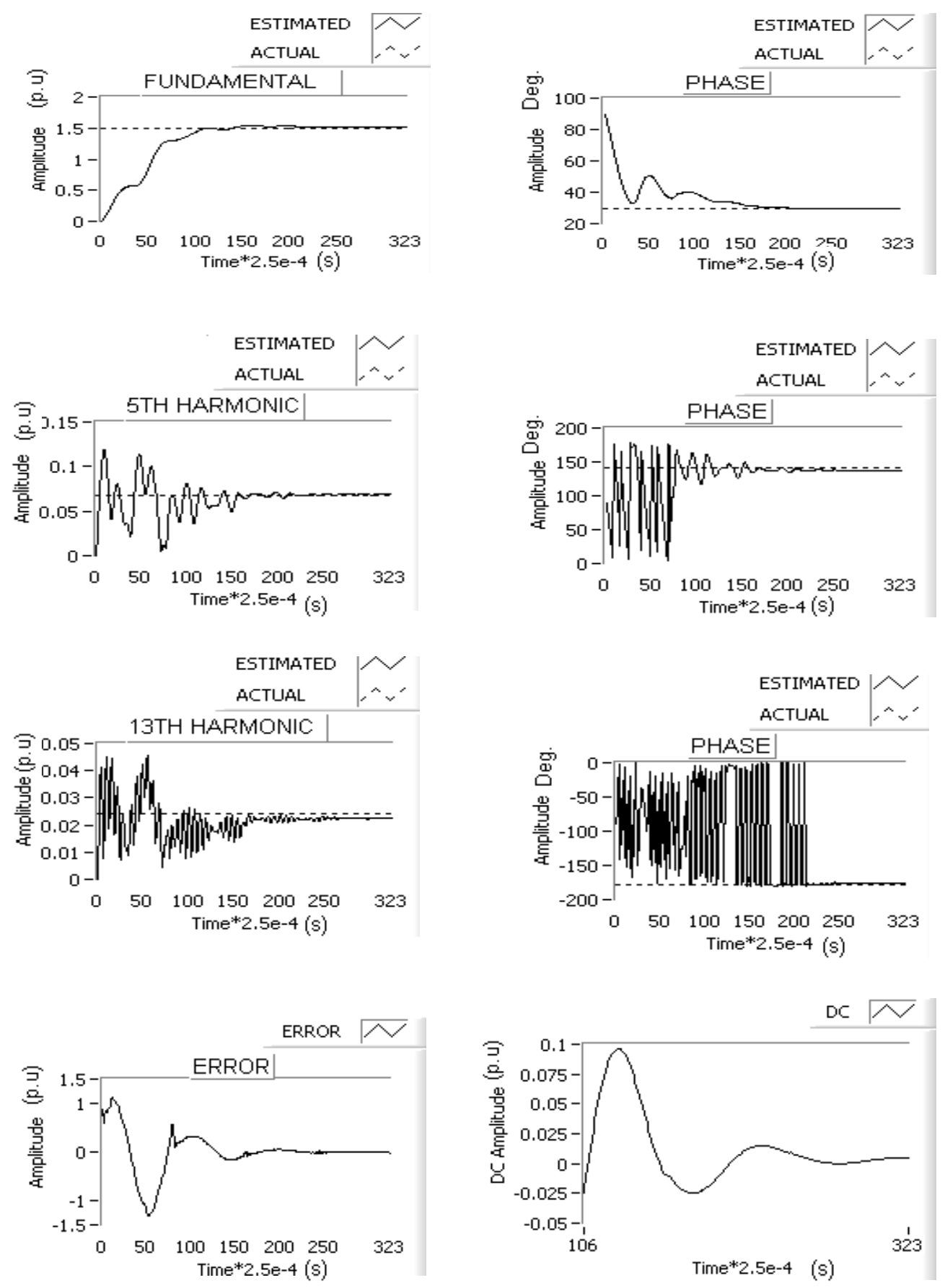

Fig. 5: Performance using ADALINE estimator with $\lambda=0.01, \alpha_{0}=0.6$ (Fixed) with initial weight vector chosen to be zero (method 1). 

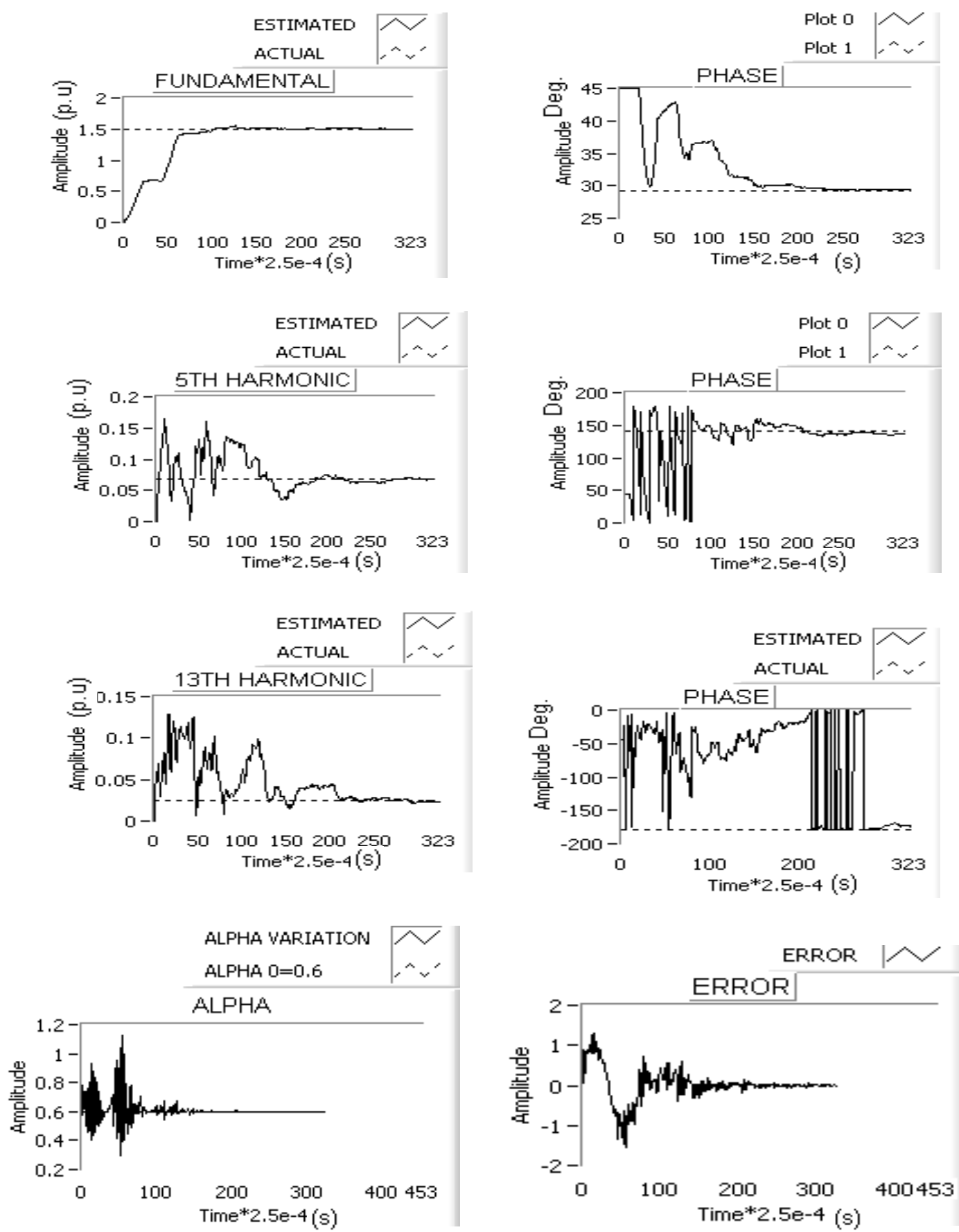

Fig. 6: Performance using ADALINE estimator with adaptive learning rate alpha using Lyapunov function $\alpha_{0}=0.6, \lambda=0.01, c_{1}=0.2, c_{2}=0.2(\operatorname{method} 2)$. 

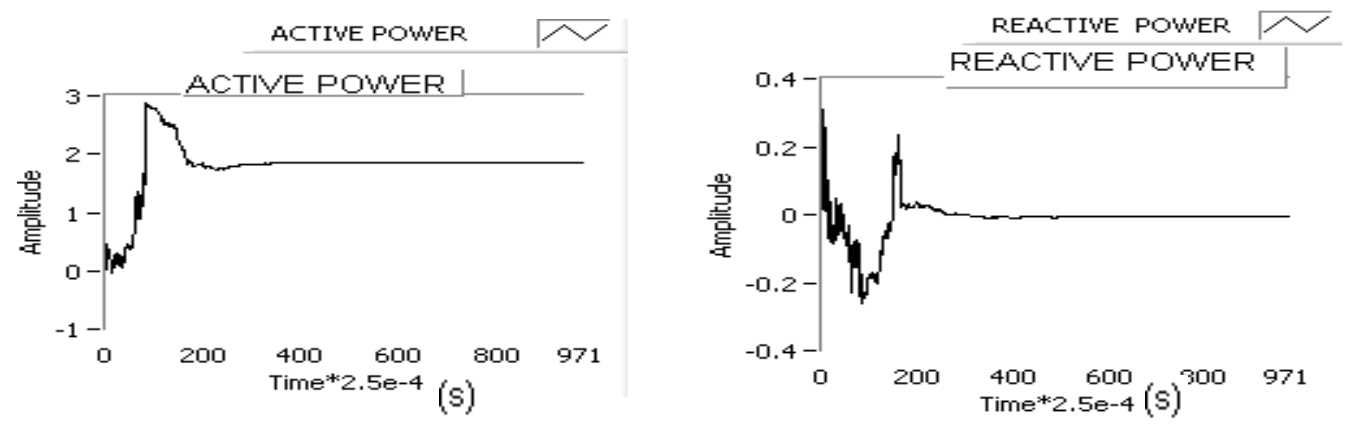

Fundamental active and reactive power
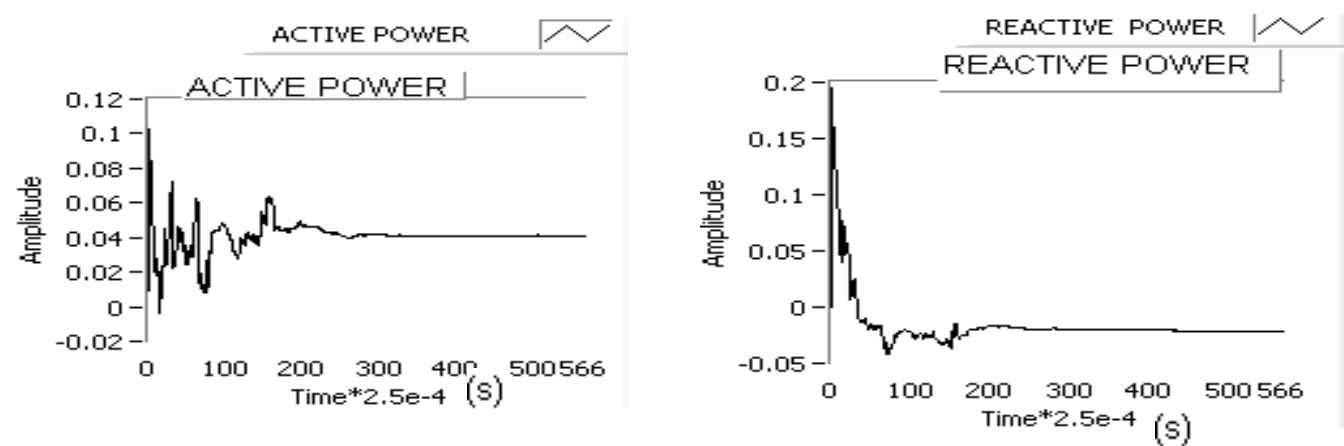

Fifth harmonic active and reactive power
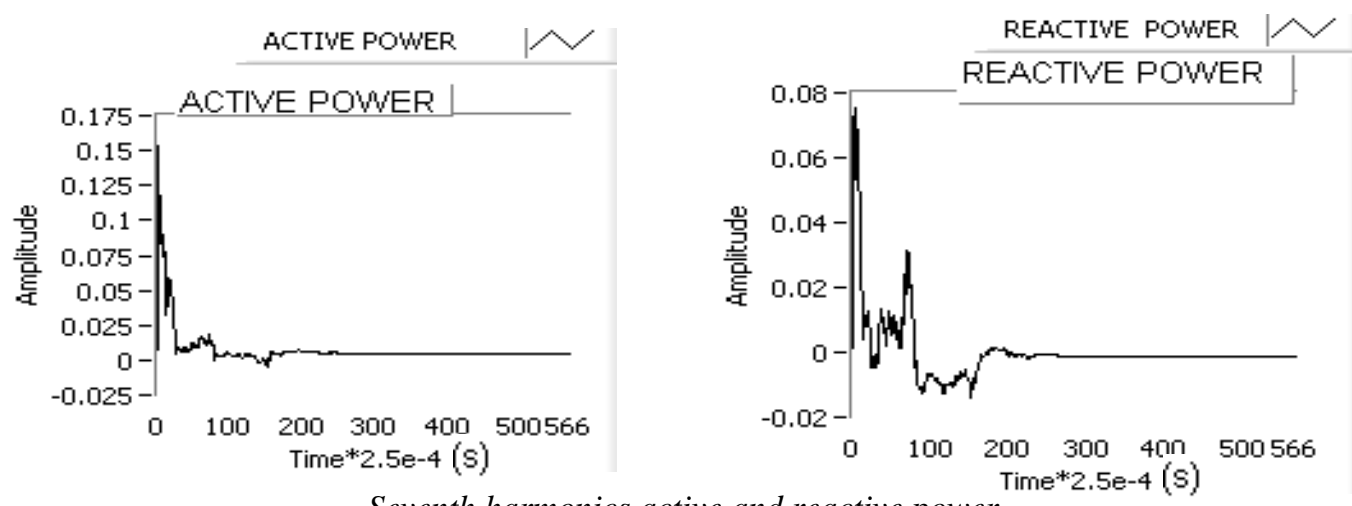

Seventh harmonics active and reactive power
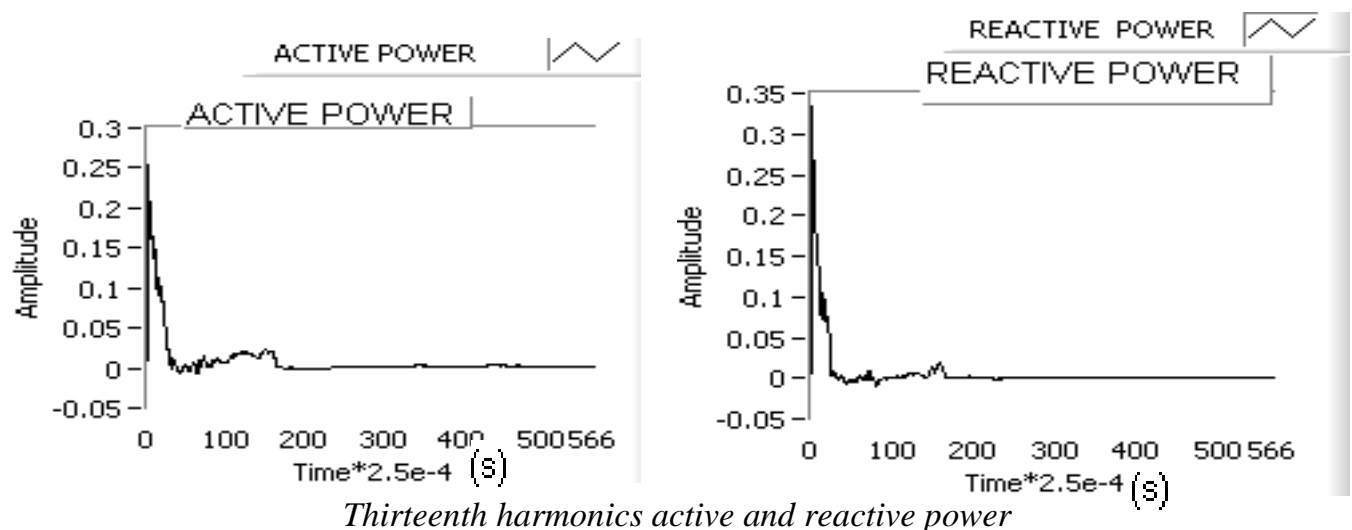

Fig. 7: Active and reactive powers of various harmonics. 


\section{CONCLUSION}

A novel measurement procedure for harmonic powers in electric power systems is presented. This method is based on the use of artificial neural networks, which increases the measurement system speed and reliability by the parallel computing architecture. The measurement system is designed to estimate the active and reactive powers of single -phase distorted system. The first module obtains the complex harmonic amplitudes of voltagelcurrent vectors. The adaline neural network for tracking the harmonic signal by illustration it is proved that the adaline neural network with Lyupunov method of changing the learning rate is more stable and quicker in convergence. Finally, the second module computes the active and reactive powers of each individual harmonic. The algorithm was applied to a nonlinear unbalanced three phase system as a practical case. The results allowed to conclude that the adopted approach is viable for the determination of the harmonic powers of the three-phase waveforms also according to an acceptable commitment between accuracy and speed.

\section{REFERENCES}

1. Arrillaga, J., Watson, N.R., and Chen, S. (2000), Power System Quality Assessment, Wiley, New York.

2. Steven Liu (1998), An adaptive kalman filter for dynamic estimation of harmonic signals, International Conference on Harmonics and Qualio of Power ICHQP '98, jointly organized by IEEEPES and NTUA,Athens, Greece, pp. 636-640. October 14-16.

3. Cichocki, A. and Lobos, T. (1994), Artificial neural networks for real-time estimation of basic waveforms of voltages and currents, IEEE Transaction on Power Systems, Vol. 9, No. 2, pp. 612-618.

4. Dash, P.K., Panda, S.K., Mishra, B., and Swain, D.P. (1997), Fast Estimation Of Voltage And Current Phasors In Powers Networks Using An Adaptive Neural Network, IEEE Transaction on Power System, Vol. 12, No. 4, pp. 1494-1499.

5. Lai, L.L., Tse, C.T., Chan, W.L., and So, A.T.P. (1999), Real-time frequency and harmonic evaluation using artificial neural networks, IEEE Transaction on Power Delivery, Vol. 14, No. 1, pp. 52-59.

6. Elmitwally, A., Abdelkader, S., and El-Kateb, M. (2000), Neural network controlled threephase four-wire shunt active power filter, Proceedings of Instrumention and Electctrical Engineering.-Generation, Transmission and Distribution, Vol. 147, No. 2, pp. 87-92.

7. Vazquez, J.R. and Salmeron, P.R. (2000), Three-phase active power filter control using neural networks, in Proc. IEEE Melecon, Vol. III, Chyprus, pp. 924-927.

8. Widrow, B. and Lehr, M. (1990), 30 years of Adaptive Neural Networks: Perceptron Madaline and Backpropagation, Proceedings IEEE, Vo1. 78, No. 9, pp. 1415-1442. 